\title{
LE NUCLÉAIRE \\ ET LES SCIENCES DU VIVANT
}

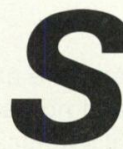

i l'énergie nucléaire est assimilée, dans l'esprit du public, à la production d'électricité - et à l'arme

atomique -, ses autres applications sont beaucoup moins connues, voire ignorées. Elles constituent pourtant de vastes champs de recherche, d'expérimentation, de pratique industrielle contribuant aux avancées des sociétés modernes. La biologie, la médecine, l'agronomie, la géologie, l'astrophysique, l'écologie... pour ne citer que ces quelques disciplines, doivent à la science nucléaire quelques-unes de leurs progrès et parfois non des moindres.

Le présent dossier illustre les apports du nucléaire aux "sciences du vivant» - la matière vivante étant définie comme une matière susceptible de métaboliser des "aliments» et de se reproduire. Ce panorama est certes incomplet - il faudrait plus d'un numéro de la RGN pour en rendre compte exhaustivement - mais il met en lumière quelques-unes des avancées importantes conduites dans le domaine du "vivant» grâce à la connaissance des rayonnements ionisants et à la maîtrise de l'utilisation des isotopes, radioactifs ou stables.

Nous remercions vivement les auteurs pour les très intéressantes contributions qu'ils ont bien voulu nous adresser. Nos remerciements vont également à Jacques Poirier et à Daniel Launois pour la part essentielle qu'ils ont prise à l'élaboration et à la coordination de l'ensemble du dossier.

Francis Sorin 